\title{
Fatigue of thin periodic triangular lattice plates
}

\author{
Yifan Li, Anton Shterenlikht, Martyn Pavier, and Harry Coules
}

Department of Mechanical Engineering, University of Bristol, Queens Building, University Walk, Bristol BS8 1TR, UK

\begin{abstract}
A method for predicting the fatigue life of triangular lattices is proposed in this paper by considering fatigue properties of single lattice struts. Fatigue tests of different sizes of lattice plates of aluminium alloy, and tests of single struts with different maximum fluctuating loads, have been conducted to validate this method. It is found that the struts in a triangular lattice break near to strut intersections, where stress and strain concentrations occur. Similar crack propagation paths were observed in different lattice plate specimens: the cracks grew at a $30^{\circ}$ angle to the initial edge crack in the upper half of lattice plate. The mixed-mode fatigue crack propagation rate was also studied and expressed using an effective stress intensity factor. A size effect on the crack growth rate of triangular lattice plates was also observed: a fatigue crack will propagate slightly quicker in larger triangular plates than in smaller ones.
\end{abstract}

\section{Introduction}

Although regular lattice structures have a high porosity, they have the advantage of high stiffness-to-weight and strength-to-weight ratios [1-2]. This kind of lightweight and materialsaving structure also has good crash energy absorption, so it can be used as protectors for lorries and space vehicle landing pads [3]. Lattice structures made of biocompatible materials, like titanium alloys, can be used for orthopaedic implants, because tissues can heal within the voids and integrate well with the implant [4].

There has been extensive research on the stiffness and strength of a wide range of 2D and 3D lattices as well as their fracture properties and damage tolerance [2, 5-9]. Recently, the fatigue behaviour of lattice structures has attracted more and more attention [10-12]. The majority of studies concern the phenomena of lattice fatigue failure or ways to improve their fatigue strength, while a few concern the fatigue properties of lattice structures and especially the methods to predict fatigue life. Wu et al. [13] investigated the influence of hot isostatic pressing (HIP) on the fatigue properties of additive manufactured Ti-6Al-4V lattice with a self-designed unit cell, which shows that the HIP treatment can improve the fatigue strength and endurance limit. Other studies show that fatigue properties of the lattice materials are highly dependent on the type of unit cell as well as on porosity [14-15]. For 3D lattice structures, higher porosities resulted in shorter fatigue lives for the same level of applied stress. Yavari et al. [16] have tried to describe fatigue failure of Ti6-Al4-V lattice structures into three-stage mechanism in detail. In the first and second stage, the strain changes slowly 
and very minimal strain will accumulate in these stages. In the final stage, the strain increases very rapidly and the specimen fails within a limited number of cycles.

However, none of these studies solve the problem of how to predict the fatigue properties of lattice structures. In this paper, a method to predict the fatigue life of $2 \mathrm{D}$ triangular lattice plates from fatigue tests of single lattice struts is proposed. The fatigue life of large lattice plate has been predicted and the macro-crack propagation rate can also be obtained from the predicted crack length-cycle curve. Fatigue tests on full lattice plates have also been conducted to validate the proposed method.

\section{Assumption and methodology}

Fatigue failure can be classified as low-cycle fatigue and high-cycle fatigue according to the number of loading cycles involved and the material's strain response during loading. Stressbased criteria are regularly used to investigate high cycle fatigue, in which the applied stress amplitude is less than the yield strength of the material [17]. Strain life estimations can be used across the entire fatigue spectrum, from low-cycle fatigue to high-cycle fatigue, which makes it an excellent method to study fatigue properties [18-20]. The sharp corners near connective knots of lattice materials will cause stress concentration and the severe stress concentration will cause plastic strain, so the plot of the fatigue life versus the strain amplitude is specific, which can be used to predict the fatigue life of lattice structures. The plastic strain-cycle $\left(\varepsilon_{p}-\mathrm{N}\right)$ curve of a fatigue test can be expressed a power law and the experimental data can be empirically fitted to a straight line, which can be written equation [21]:

$$
\frac{\Delta \varepsilon_{p}}{2}=\varepsilon_{f}^{\prime}\left(2 N_{f}\right)^{c}
$$

where $c$ is the fatigue ductility exponent and $\varepsilon_{f}^{\prime}$ is the fatigue ductility coefficient (the sign of $c$ is negative). If we know the parameters $\varepsilon_{f}^{\prime}$ and $c$ in the equation (1), which depend on material properties, then the fatigue cycles under specific strain can be calculated using this formula.

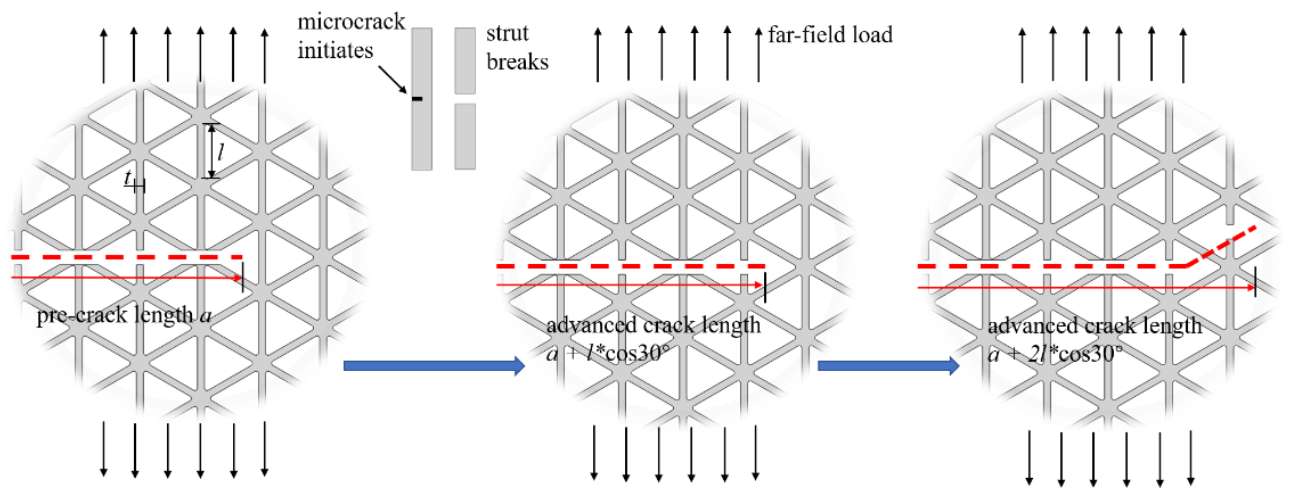

Fig. 1. The mechanism of crack propagation in a 2D triangular lattice. Cracks existing with a single strut are referred to as microcracks, while a macrocrack propagates through the lattice.

A crack will firstly initiate in a single strut of the lattice when it under cyclic loading, then the crack propagates to the critical length for fast fracture. At this time, the strut breaks and the macrocrack of lattice structure can be seemed as advancing by a unit cell. Then it needs the strut near the crack tip to initiate a crack again, which means that lattice structures could have a good resistance to fatigue, because crack initiation life usually occupies large 
proportion of whole fatigue life. In our analysis, the crack tip is assumed to be at the centre of the cell rather than at the unbroken strut that adjacent the crack, which is shown in Fig.1.

At the same time, cyclic load will also accumulate damage in struts that are not adjacent the crack tip, and this phenomenon can be described by Miner's rule [22] in the formula expressed as:

$$
\sum_{i} \frac{N_{i}}{N_{i f}}=1
$$

where $i$ is the order of the specific stress applied to the strut, $N_{i}$ is the number of actual running cycles at a certain stress and $N_{i f}$ is the number of cycles to failure. A strut will break when the accumulation damage in formula (2) caused by different cyclic loads equal to 1 . The numbers of cycles for the different struts to fail are summed to calculate a lifetime prediction for the whole lattice.

In a triangular lattice, the stress state predominantly involves stretching of the struts: the bending stress is only a few percent of the axial stress at the failure site [5-6]. Our method assumes that these negligible bending stresses have little effect on each struts' fatigue life. We also assume that struts within the lattice have identical behaviour to single struts tested individually in tensile-tensile fatigue tests.

The prediction approach can be described using the following steps:

(1) Perform fatigue tests of single struts cut from lattice plates and obtain the $\varepsilon_{p}-\mathrm{N}$ curve as well as the strain-based formula of struts under tension-tension cyclic load.

(2) Obtain the stress-strain state of lattice plate under specific load using Finite Element Analysis (FEA), and use the $\varepsilon_{p}$-N curve of single struts with the formula to predict the overall fatigue life of a strut in the lattice near the crack tip.

(3) Considering cumulative damage caused by previous loading, use Miner's rule to calculate the actual fatigue endurance of this crack-tip strut.

(4) Delete the failed strut near crack tip and extend the macro-crack, then repeat steps (2) to (4) until the lattice plate is predicted to fail in fast fracture.

\section{Experimental procedure and results}

All the lattice plates are cut from the $2 \mathrm{~mm}$ thick aluminium alloy 1050A sheet by using a Flow Mach2c waterjet cutter. Waterjet cutting prevents the temperature of material from increasing during the manufacturing procedure, which will ensure manufacturing accuracy. 120 grade ruby dust with particle sizes of 106 to $250 \mu \mathrm{m}$ was used in the machine, and this still caused a rough surface on the lattice struts. The designed width of the cell wall in lattice plates is $1 \mathrm{~mm}$ and the nominal value of cell wall length is $10 \mathrm{~mm}$. For fatigue testing of single structs, structs were extracted from lattice plates and glued with additional plates sandwiching the ends to improve clamping, as shown in Fig. 2.

A servo-hydraulic testing machine (Instron 8872) with a maximum sensor load capacity of $1 \mathrm{KN}$ was used in single struts' fatigue test. The frequency of fatigue loading was $10 \mathrm{~Hz}$, and the load ratio $R$ was equal to 0.1 for all tests. All of the single strut specimens broke between the glue points and near to struts intersections, which means the clamping method did not influence the fatigue properties measured from the specimen.

Six small lattice plates were used to test the fatigue life of lattices with three different widths. Three groups of plates were used with three lengths of initial edge crack, the cracks were cut through 2,3 , and 4 cells in the plates, respectively. The relative crack length $\alpha=\mathrm{a} / \mathrm{w}$ in three groups of plates was $0.163,0.235$ and 0.252 (see Figure 2). Test conditions are the same as the test of single struts except that the servo-hydraulic testing machine (Instron 8872) 
used a maximum load capacity $10 \mathrm{KN}$ sensor. To validate the applicability of this method on lattice plates, different loads were used on different lattice plates.

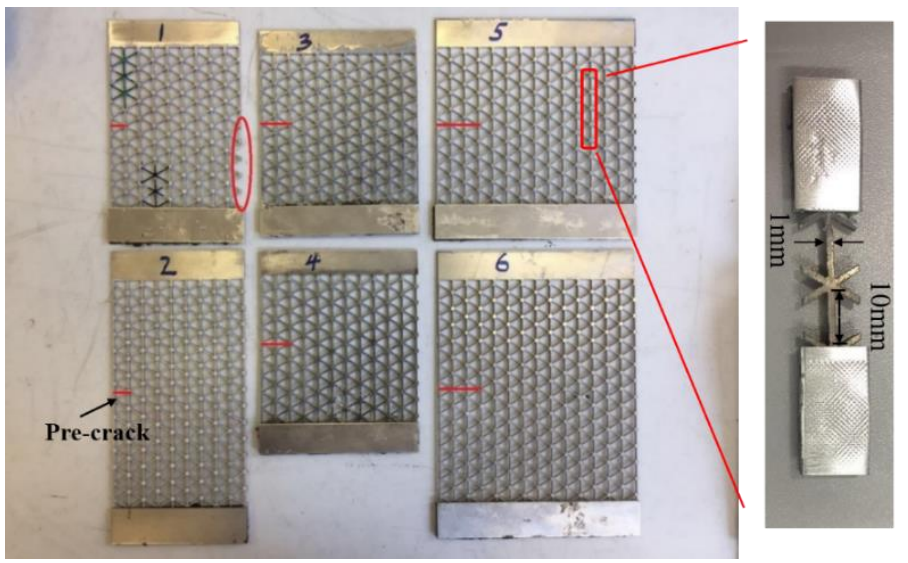

Fig. 2. Several differently-sized lattice plates and a single-strut specimen ready for fatigue testing. The red horizontal lines in the lattice plates indicate the initial crack length.

The test machine was stopped when one strut near crack tip broke to record the cycles, then started the machine again to run the test. The whole fatigue life of the plate is the sum of fatigue lives of different single struts. The fatigue cycles of first two struts occupied a large proportion of the whole plates' fatigue life. The fatigue cycles of 4 specimens in total were recorded well. It was observed that only vertically-aligned struts will break when the plates under cyclic loading. Therefore, the fatigue crack path in all lattice plates had a specific pattern: the crack developed in the direction $30^{\circ}$ to the horizontal axis of the initial edge crack in the upper half plate. This is identical to the crack propagation behaviour of similar lattices under monotonic fracture conditions [9].

\section{Method validation and comparison}

FEA was used to determine the stress and strain state of single struts under loading and obtain the $\varepsilon_{p}$-N curve according to Equation (1), then use the proposed method to predict the fatigue life of the lattice plates. The single lattice strut glued with four small aluminium plates are modelled as one part. The material stress-strain curve is shown in Fig.3. [23]. Three dimensional (3D) finite element models were used to simulate the strut tension with reducedintegration 8-noded brick (Abaqus type C3D8R) and 10-noded tetrahedral (C3D10) elements. A higher mesh density was used near to the sharp corners of the strut joints, while elements far from the corners had a coarser mesh to reduce the simulation time. In this simulation, the bottom part of single strut was set to be fixed in all directions and the top part can only move along the loading direction.

After the simulation of fatigue test, the experimentally-observed strut fatigue life was related to the specific plastic strain amplitude from the FEA. The fitting curve is shown in Fig. 4. The two material constants were found by using formula (1): $\varepsilon_{f}^{\prime}=1.89810 /$ cycle, $c=-$ 0.63859 .

The boundary conditions used for FEA of complete lattice plates were the same as the fatigue tensile test, and the stress and strain states of struts near the crack tip was obtained. The plastic strain was substituted into the equations and obtain a prediction of the fatigue endurance. The struts with largest plastic strain were deleted, extending the fatigue crack, and these steps were repeated until lattice failure. The simulated crack path was the same as 
observed in the fatigue experiments, shown in Fig.5, which is in the direction $30^{\circ}$ to the horizontal axis of initial edge crack. The comparison of predictions of the proposed method and experimental results is shown in Fig. 6, and they agree very well.

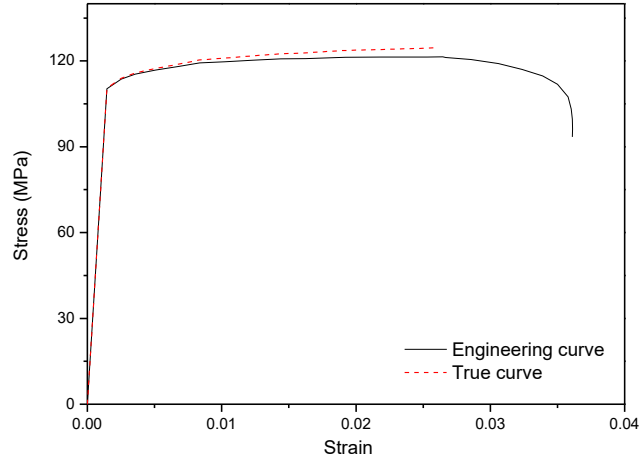

Fig. 3. Stress-strain curve of aluminium 1050A

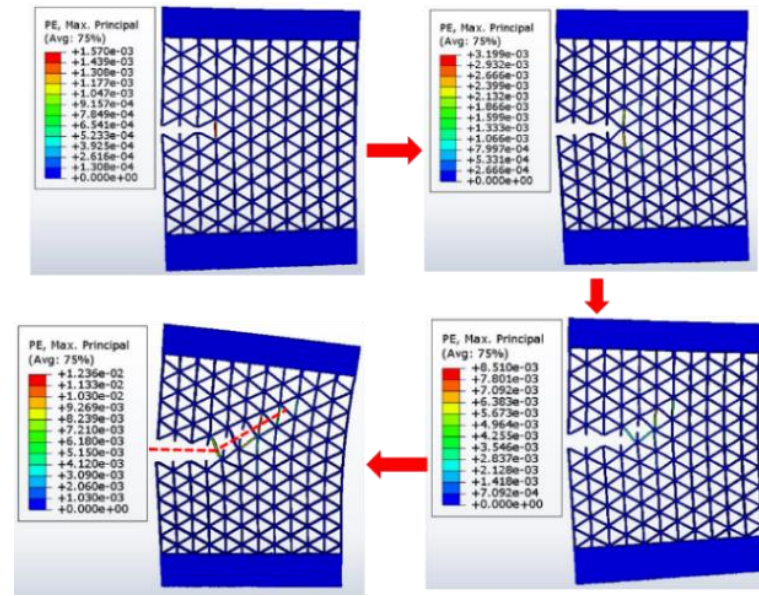

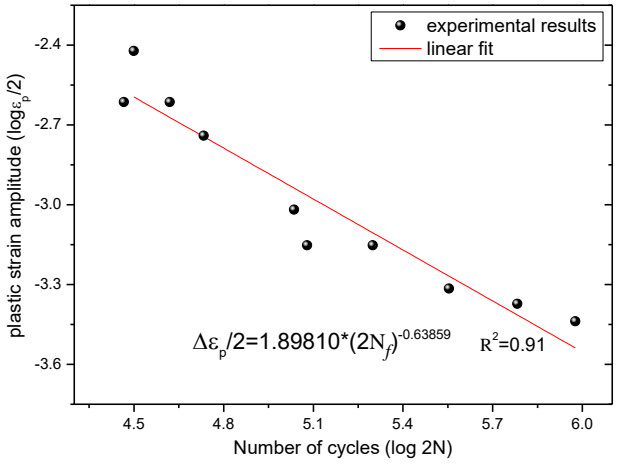

Fig. 4. Fitted curve of single struts' fatigue data

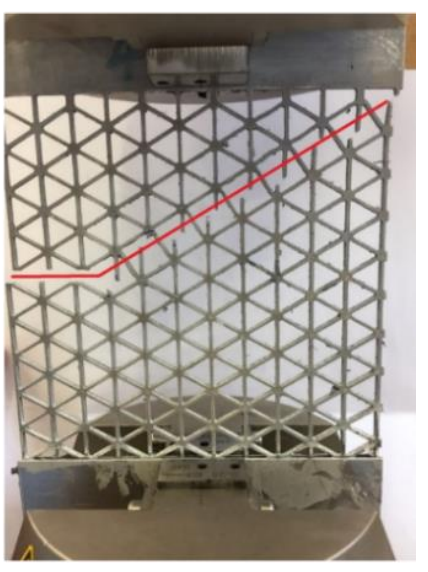

Fig. 5. Fatigue procedure and crack path in specimen 4

In most cases, a greater fatigue endurance is predicted than was experimentally, because some damage was introduced into the lattice struts during processing. Most of the width of struts' cell walls are also less than the design width $1 \mathrm{~mm}$. For example, the width of specimen 2, 5 and 6 are $0.79 \sim 0.92 \mathrm{~mm}, 0.76 \sim 0.89 \mathrm{~mm}$ and $0.73 \sim 0.91 \mathrm{~mm}$, respectively. While the struts' width of specimen 4 is $1.06 \sim 1.14 \mathrm{~mm}$, and its test result also larger than the predicting cycles.

Fatigue life predictions for specimen 4 and 6 agree well with test results, and the relative errors are $7 \%$ and $13.5 \%$, which are really insignificant considering that there will be scatter in material properties, experimental errors as well as the damage introduced during processing of waterjet cutting. The error between experimental result and predicting result of single strut in lattice plate is written as $e_{i j}$, which mean it is the error of strut $j$ in plate $i$. The error between test results and predictions in plate 2 and 5 is shown in Table 1 . It is obvious that the total error of whole plate is mainly caused by one single strut like strut 1 in plate 2 and strut 2 in plate 5. Actually, the fatigue cycles of struts in specimen 2 also have acceptable fatigue life predictions, and the large error is caused by strut 1 . We hypothesize strut 1 survived a very small number of cycles because it was damaged before the test. 


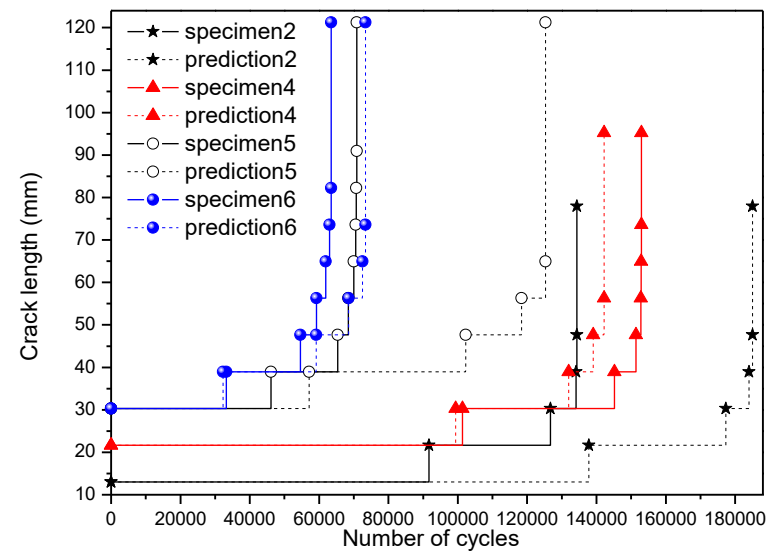

Fig. 6. Comparison between the predictions and experimental results

Table 1 Error between experimental results and predictions of struts in plate 2 and 5

\begin{tabular}{|c|c|c|c|c|c|}
\hline $\begin{array}{c}\text { Plate } \\
\text { number }\end{array}$ & Strut number & Test results (N) & Prediction (N) & Error & $e_{i j} / \sum_{j=1}^{4} e_{i j}$ \\
\hline \multirow{3}{*}{ Plate 2 } & 1 & 91672 & 137788 & 46116 & $88.60 \%$ \\
\cline { 2 - 6 } & 2 & 35012 & 39514 & 4502 & $8.65 \%$ \\
\cline { 2 - 6 } & 3 & 7405 & 6712 & 693 & $1.33 \%$ \\
\cline { 2 - 6 } & 4 & 229 & 966 & 737 & $1.42 \%$ \\
\hline \multirow{3}{*}{ Plate 5 } & 1 & 46114 & 57111 & 10997 & $21.41 \%$ \\
\cline { 2 - 6 } & 2 & 19283 & 45167 & 25884 & $50.39 \%$ \\
\cline { 2 - 6 } & 3 & 3018 & 13870 & 10852 & $21.13 \%$ \\
\cline { 2 - 6 } & 4 & 1599 & 5231 & 3632 & $7.07 \%$ \\
\hline
\end{tabular}

\section{Prediction of crack growth rate}

Since lattice structures used in practice usually cover large areas or have a large number of cells, it is necessary to study the fatigue response of huge lattice plates. Gu et al. [9] performed experiments on triangular lattice plates and showed that a ratio of specimen width to unit cell size of 24 can mimic the response of an infinitely-extended lattice plate. The lattice plate in this paper used to predict fatigue life has 27 cells in length and 24 cells in width. The boundary conditions in this prediction are the same as the small lattice plates we tested before, and the maximum fatigue loads were $3600 \mathrm{~N}, 3300 \mathrm{~N}$ and $3100 \mathrm{~N}$, respectively. The fatigue life predictions for large lattice plates are similar to the small plates experimental results, the first and second struts' fatigue life occupies a large portion of the whole plates' fatigue lives.

Fatigue crack growth rate is an important parameter to describing the resistance of materials to fatigue. Paris related the stress intensity factor range $(\Delta \mathrm{K})$ to crack growth under a fatigue stress regime and proposed the Paris law [24]:

$$
\begin{gathered}
\frac{d a}{d N}=\mathrm{C} \Delta K^{m} \\
\Delta K=K_{\text {max }}-K_{\text {min }}
\end{gathered}
$$

where $C$ and $m$ are material-dependant constants, $a$ is the crack length and $N$ is the number of fatigue cycles. 
The fatigue crack growth rate and SIF range were calculated according to the secant method suggested in the ASTM E647 [25], which are shown as:

$$
\begin{gathered}
\frac{d a}{d N}=\frac{a_{j+1}-a_{j}}{N_{j+1-N_{j}}} \\
\Delta K=\frac{1}{2}\left(\Delta K_{j}+\Delta K_{j+1}\right)
\end{gathered}
$$

Although the fatigue loads used were perpendicular to the initial crack, the fatigue crack propagated in inclined direction, so I/II mixed mode fracture will happen in triangular lattice plates. Effective stress intensity factor $K_{\text {eff }}$ is frequently used to study mixed mode fracture failure and fatigue failure [26]:

$$
K_{e f f}=\sqrt{K_{I}^{2}+K_{I I}^{2}}
$$

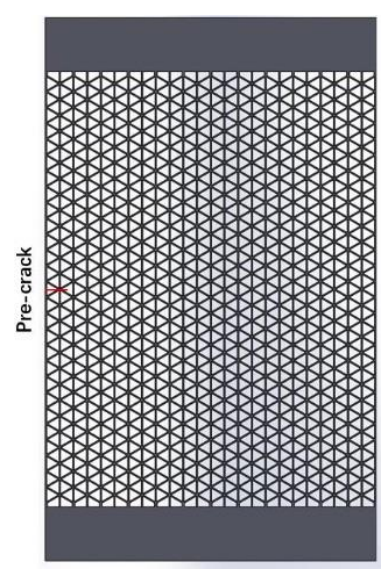

Fig. 7. Huge lattice plate to predict fatigue life

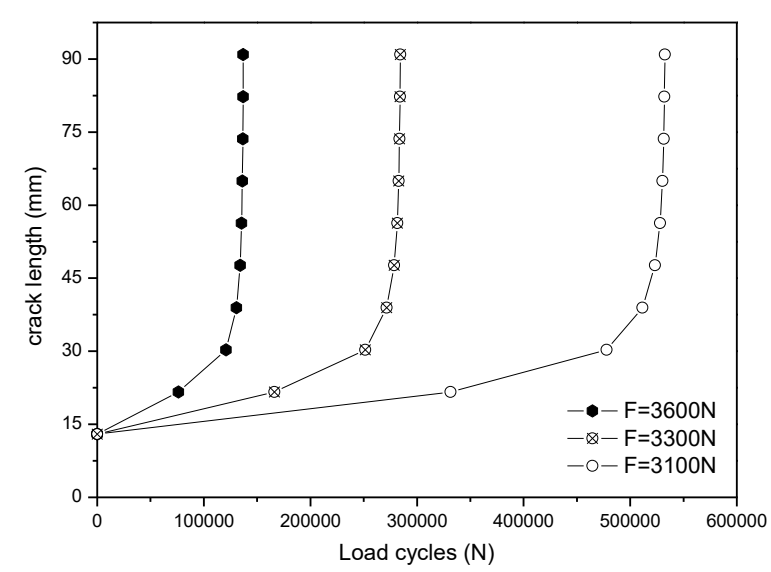

Fig. 8. Predicting fatigue life of large plates

The SIF of lattice plates was calculated by using FEA with the homogenization method. The effective elastic properties of lattice material are related to the parent material and topology. The effective Young's modulus and Poisson's ratio can be calculated from the following formula [9]:

$$
\begin{gathered}
E_{e}=2 \sqrt{3} t_{0} \frac{1+t_{0}^{2}}{3+t_{0}^{2}} E_{s} \\
v_{e}=\frac{1-t_{0}^{2}}{3+t_{0}^{2}} \\
t_{0}=\frac{t}{l}
\end{gathered}
$$

Where $E_{e}$ and $v_{e}$ are the effective Young's modulus and Poisson's ratio of triangular lattice, respectively. $E_{s}$ is the Young's modulus of parent material, which can be obtained from the stress-strain curve. The nominal length $l$ of strut is $10 \mathrm{~mm}$, and nominal thickness $t$ is $1 \mathrm{~mm}$.

The crack growth rates of small lattice plates and predictions for large lattices are shown in Fig. 9. In general, cracks in large plates propagate more rapidly than the test small specimens, because the relative length of cracks in small plates increase significantly, which will cause large stress intensity factors calculated in FEA. In reality, the dimensions of initial cracks comparing with the real-life lattice structures should be insignificant, and the relative crack length increases with the crack tip extension should also be minor. Therefore, the 
predicted crack growth rate in large lattice plates can better represent triangular lattice material comparing with results from tests using small specimens. The two material constants of for a large plate from Equation (3) were found to be: $C=2.6534 \times 10^{-13} \mathrm{~mm} / \mathrm{cycle}$, $m=4.9157$.

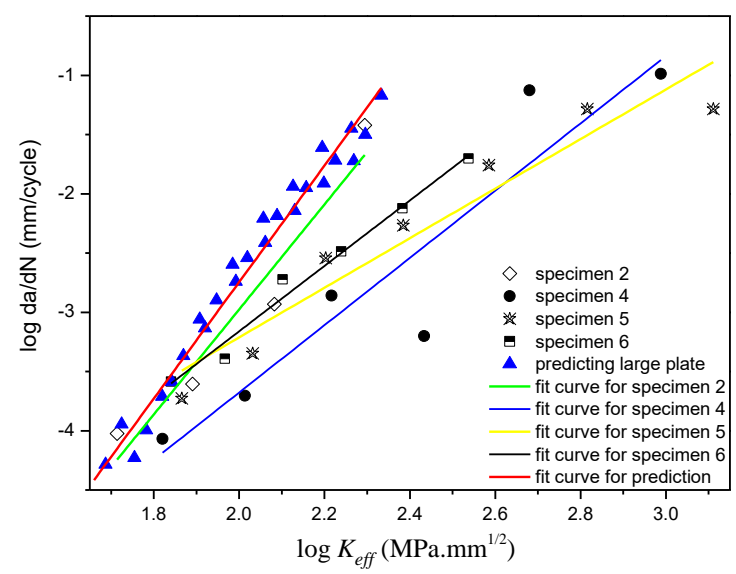

Fig. 9. Fatigue crack growth rates of different plates

\section{Conclusion}

In this study, a method was proposed for predicting the fatigue life of 2D triangular lattices, and was found to perform very well. Predictions of the crack path obtained using this method were also consistent with experiments: in a lattice of this type develops in the $30^{\circ}$ direction. The fatigue crack growth rate depends on size of the lattice structure, and so small lattice plates the predicted crack growth rate was slightly slower observed experimentally. The crack growth rate of a large plate can better represent lattice structure in real life considering that real lattice structures generally contain a large number of cells.

\section{References}

1. L.J. Gibson, M.F. Ashby, Cellular solids: structure and properties, Cambridge university press (1999)

2. A.J. Wang, D.L. McDowell. In-plane stiffness and yield strength of periodic metal honeycombs. J Eng Mater-T ASME, 126(2): 137-156 (2004)

3. S. Prabhu, V.K. Rajan, R. Nikhil. Applications of Cellular Materials-An Overview. Appl Mech Mater, 766: 511-517 (2015)

4. F. Li, J. Li, T. Huang, H. Kou, L. Zhou. Compression fatigue behavior and failure mechanism of porous titanium for biomedical applications. J Mech Behav Biomed Mater, 65: 814-823 (2017)

5. N.A. Fleck, X.M. Qiu. The damage tolerance of elastic-brittle, two-dimensional isotropic lattices. J Mech Phys Solids, 55(3): 562-588 (2007)

6. X. Qiu, L. He, Y. Qian, X. Zhang. Plastic zone of semi-infinite crack in planar kagome and triangular lattices. Acta Mech Solida Sin, 22(3): 213-225(2009)

7. H.C. Tankasala, V.S. Deshpande, N.A. Fleck. Crack-Tip Fields and Toughness of TwoDimensional Elasto-Plastic Lattices. J Appl Mech-T ASME, 82(9): 1-10 (2015) 
8. C. Chen, T.J. Lu, N.A. Fleck. Effect of imperfections on the yielding of two-dimensional foams. J Mech Phys Solids, 47(11): 2235-2272 (1999)

9. H. Gu, M. Pavier, A. Shterenlikht. Experimental study of modulus, strength and toughness of 2D triangular lattices. Int J Solids Struct, 152: 207-216 (2018)

10. O.B. Olurin, K.Y.G. McCullough, N.A. Fleck, M.F. Ashby. Fatigue crack propagation in aluminium alloy foams. Int J Fatigue, 23(5): 375-382 (2001)

11. A. Shipsha, M. Burman, D. Zenkert. On mode I fatigue crack growth in foam core materials for sandwich structures. J Sandw Struct Mater, 2(2): 103-116 (2000)

12. D. Zenkert, M. Burman. Fatigue of closed cell foams. J Sandw Struct Mater, 13(4): 467478 (2011)

13. M.W. Wu, J.K. Chen, B.H. Lin, P.H. Chiang. Improved fatigue endurance ratio of additive manufactured Ti-6Al-4V lattice by hot isostatic pressing. Mater. Des, 134: 163170 (2017)

14. S.A. Yavari, S.M. Ahmadi, R. Wauthle, B. Pouran, J. Schrooten, H. Weinans, A.A. Zadpoor. Relationship between unit cell type and porosity and the fatigue behavior of selective laser melted meta-biomaterials. J Mech Behav Biomed Mater, 43: 91-100 (2015)

15. S. Zhao, S.J. Li, W.T. Hou, Y.L. Hao, R. Yang, R.D.K Misra. The influence of cell morphology on the compressive fatigue behavior of Ti-6Al-4V meshes fabricated by electron beam melting. J Mech Behav Biomed Mater, 59: 251-264 (2016)

16. S.A. Yavari, R. Wauthlé, J. Stok, et al. Fatigue behaviour of porous biomaterials manufactured using selective laser melting. Mater. Sci. Eng. C., 33(8): 4849-4858 (2013)

17. M.F. Ashby, D.R.H. Jones, Engineering materials 1: an introduction to properties, applications and design. Elsevier (2012)

18. T.J. Spradlin, R.V. Grandhi, K. Langer. Experimental validation of simulated fatigue life estimates in laser-peened aluminium. Int. J. Struct. Integr., 2(1): 74-86 (2011)

19. G. Fajdiga, M. Sraml. Fatigue crack initiation and propagation under cyclic contact loading. Eng Fract Mech, 76(9): 1320-1335 (2009)

20. Fe-safe, Fatigue theory reference manual-Volume 2. Safe Technology Limited (2017)

21. W. Cui. A state-of-the-art review on fatigue life prediction methods for metal structures. J Mar Sci Technol, 7(1): 43-56 (2002)

22. M. Miner. Cumulative damage in fatigue. J Appl Mech-T ASME, 67: A159-164 (1945)

23. F. Gharbi, S. Sghaier, H. Hamdi, T. Benameur. Ductility improvement of aluminum $1050 \mathrm{~A}$ rolled sheet by a newly designed ball burnishing tool device. Int $\mathrm{J}$ Adv Manuf Tech, 60(1-4): 87-99 (2012)

24. P. Paris, F. Erdogan. A critical analysis of crack propagation laws. J Basic Eng-T ASME, 85(4): 528-533 (1963)

25. ASTM, Standard test method for measurement of fatigue crack growth rates, in: E647 15, United States (2015)

26. S.B. Biner. Fatigue crack growth studies under mixed-mode loading. Int J Fatigue, 23: 259-263 (2001) 